\title{
Phase I dose-escalation study of helical intensity-modulated radiotherapy-based stereotactic body radiotherapy for hepatocellular carcinoma
}

\author{
Jun Won Kim¹, Jinsil Seong ${ }^{2}$, Ik Jae Lee ${ }^{1}$, Joong Yeol Woo ${ }^{2}$, Kwang-Hyub Han ${ }^{3}$ \\ ${ }^{1}$ Department of Radiation Oncology, Gangnam Severance Hospital, Yonsei University College of Medicine, Seoul, Korea \\ ${ }^{2}$ Department of Radiation Oncology, Yonsei Cancer Hospital, Yonsei University College of Medicine, Seoul, Korea \\ ${ }^{3}$ Department of Internal Medicine, Severance Hospital, Yonsei University College of Medicine, Seoul, Korea \\ Correspondence to: Jinsil Seong, email: jsseong@yuhs.ac
}

Keywords: stereotactic body radiotherapy, hepatocellular carcinoma, dose escalation, intensity-modulated radiotherapy

Received: March 20, 2016

Accepted: April 16, 2016

Published: May 18, 2016

\section{ABSTRACT}

Background: Phase I trial was conducted to determine feasibility and toxicity of helical intensity-modulated radiotherapy (IMRT)-based stereotactic body radiotherapy (SBRT) for hepatocellular carcinoma (HCC).

Results: Eighteen patients ( 22 lesions) were enrolled. With no DLT at 52 Gy (13 Gy /fraction), protocol was amended for further escalation to $60 \mathrm{~Gy}$ (15 Gy/fraction). Radiologic complete response rate was $\mathbf{8 8 . 9 \%}$. Two outfield intrahepatic, 2 distant, 4 concurrent local and outfield, and 1 concurrent local, outfield and distant failures (no local failure at dose levels 3-4) occurred. The worst toxicity was grade 3 hematologic in five patients, with no gastrointestinal toxicity $>$ grade 1 . At median follow-up of 28 months for living patients, 2-year local control, progression-free (PFS), and overall survival rates were $71.3 \%, 49.4 \%$ and $69.3 \%$, respectively. Multi-segmental recurrences prior to SBRT was independent prognostic factor for PFS $(p=0.033)$.

Materials and Methods: Eligible patients had Child-Pugh's class A or B, unresectable HCC, $\leq 3$ lesions, and cumulative tumor diameter $\leq 6 \mathrm{~cm}$. Starting at $36 \mathrm{~Gy}$ in four fractions, dose was escalated with $2 \mathrm{~Gy} /$ fraction per dose-level. CTCAE v $3.0 \geq$ grade 3 gastrointestinal toxicity and radiation induced liver disease defined dose-limiting toxicity (DLT).

Conclusions: Helical IMRT-based SBRT was tolerable and showed encouraging results. Confirmatory phase II trial is underway.

\section{INTRODUCTION}

The most common etiology for hepatocellular carcinoma (HCC) in Korea is viral infection (hepatitis $\mathrm{B}$ and $\mathrm{C}$ ), and a large proportion of newly diagnosed HCC accompany advanced cirrhosis [1]. Surgical resection and percutaneous ablation can provide longterm overall survival [2]; however, eligibility for these curative procedures is limited by preexisting conditions including hepatic dysfunction, tumor number and size, and vascular invasion. Stereotactic body radiotherapy (SBRT) has shown high rates of local control for primary and metastatic liver cancers [3-5]. In SBRT, a biologically equivalent dose $>100$ Gy is delivered using highly conformal, hypofractionated radiation in 2-5 fractions [6], and this technique requires precision targeting and reproducibility in treatment setting. Given the steep dose fall-off and reduced number of fractions, the risk of local failure and normal tissue injury due to geometric miss is high with SBRT. Thus, major challenges in achieving safe and accurate SBRT for intrahepatic tumors include defining and limiting respiratory liver motion during treatment and providing accurate daily image-guidance.

Helical Tomotherapy (HT) (Accuray, Madison, WI) is well-suited for delivering SBRT. The use of up to 51 beam angles for treatment planning allows for highly conformal dose distributions with improved sparing of normal tissues compared to more conventional 3D plans [7]. HT-based SBRT is often utilized for treating brain [8] or spine lesions [9]; however, its role in treating 
intrahepatic tumors has not been explored. HT is effective in treating multiple targets simultaneously, and we have previously shown its adequacy for multiple metastatic tumors [10]. The ability of HT to target multiple lesions simultaneously has been demonstrated for intrahepatic tumors [11] as well, and this is an important ability for liver SBRT, because most studies allowed patients with up to three intrahepatic lesions for eligibility $[12,13]$.

Image guidance for HT is provided via CT detector mounted opposite the radiation source, which is used for megavoltage CT (MVCT) imaging [14]. A common problem in using MVCT for image guidance is the low contrast resolution observed with the MVCT imaging system relative to kilovoltage $(\mathrm{kV}) \mathrm{CT}$ [15], particularly when localizing treatment sites in the abdomen. However, the contour of the entire liver can be a surrogate, and rigid liver-to-liver registration using MVCT in combination with respiration control can be an effective imageguidance system for liver tumors.

We have been using HT with MVCT image guidance for treating locally advanced HCC at our institution since 2006 [16]. We report the results of a phase I dose escalation trial that was designed to determine the feasibility and toxicity of helical intensity-modulated radiotherapy (IMRT)-based SBRT for primary HCC.

\section{RESULTS}

\section{Patients}

From March 2012 to April 2014, 18 patients with 22 lesions were enrolled. Table 1 shows the demographic and treatment data. All patients had Child-Turcotte-Pugh (CTP) A (score 5 in 17 patients and 6 in one patient), and no patient had portal vein tumor thrombosis. The median cumulative tumor diameter was $2.05 \mathrm{~cm}$ (range $1.0-4.4 \mathrm{~cm}$ ). The dose was initially escalated to $52 \mathrm{~Gy}$ (13 Gy/fraction) without DLT. The protocol was amended for a further escalation to $60 \mathrm{~Gy}$ (15 Gy/fraction). The total number of patients analyzed in this study included additional patients enrolled in dose levels 1 and 3 while the amended protocols were being approved. Table 2 shows the dosimetric parameters from the radiotherapy planning. The median value for PTV was $79.9 \mathrm{cc}$ (8.16 to $225.3 \mathrm{cc})$. The median normal liver volume was $1124 \mathrm{cc}$ (801 to $1736 \mathrm{cc}$ ), and the median value of the mean dose to normal liver was $9.7 \mathrm{~Gy}$ (3.0 to $14.3 \mathrm{~Gy})$.

\section{Toxicity}

SBRT was well tolerated, with no DLT observed at any level. Table 3 shows toxicities that worsened from pre-SBRT (baseline) conditions, within the first 3 months after SBRT or prior to salvage treatment in case of treatment failure. The worst liver toxicity was grade 2 hyperbilirubinemia in one patient, and no GI toxicity greater than grade 1 occurred.
Grade 3 leukocytopenia and thrombocytopenia developed in two and five patients, respectively. Six of these patients had grade 2 and one had grade 1 hematologic events prior to SBRT. Grade 2 radiation pneumonitis occurred in one patient with $\mathrm{HCC}$ in segment 8.

\section{Response rate and tumor control}

A radiologic CR was achieved in 16 patients $(88.9 \%)$ with a median time to a radiologic CR of 6.0 months (range 0.7-12.3 months). One patient at dose level 1 achieved a pathologic CR, which was confirmed at liver transplantation 14 months after SBRT. Nine patients experienced disease progression: 2 outfield intrahepatic, 2 distant, 4 local and outfield, and 1 local, outfield and distant failures (Figure 1). At dose level 1, one outfield intrahepatic failure, 1 concurrent local and outfield, and 1 outfield failure followed by local failure were observed. At dose level 2, one local failure followed by outfield failure and 1 lung metastasis were observed. At dose level 3 , one outfield failure and 1 lung metastasis were observed, and another patient experienced outfield failure followed by local failure which was due to tumor progression into the treated area. At dose level 4, one patient experienced concurrent outfield failure and lung metastasis followed by local failure which was due to rapid tumor progression into the treated area.

\section{Survival and prognostic factors}

At a median follow-up of 23 months (range 11-38 months) for all patients and 28 months (range 13-38 months) for living patients, the 1- and 2-year local control rates were $77.8 \%$ and $71.3 \%$, outfield intrahepatic progression-free survival rates were $61.1 \%$ and $61.1 \%$, distant metastasis-free survival rates were $88.9 \%$ and $83.0 \%$, progression-free survival (PFS) rates were $55.6 \%$ and $49.4 \%$, and overall survival rates were $94.4 \%$ and 69.3\%, respectively (Figure 2A and 2B). Table 4 shows the results of univariate and multivariate analyses of the clinical factors influencing PFS. Multi-segmental recurrences prior to SBRT showed a significant correlation with poor PFS rates in univariate $(p=0.029)$ and multivariate analyses $(p=0.033)$. PFS and OS of the patients with $(n=10)$ and without multisegment recurrences $(n=8)$ are shown in Figure $2 \mathrm{C}$ and $2 \mathrm{D}$. Seven of the 10 patients with multi-segmental recurrences showed failures after SBRT: 1 local, 4 outfield intrahepatic, 1 lung metastasis, and 1 synchronous outfield intrahepatic and lung metastasis.

\section{DISCUSSION}

Challenges in treating intrahepatic tumors with RT include defining and limiting respiratory liver motion, accurate delineation of hypovascular tumors, minimizing 
Table 1: Demographic and treatment data $(n=18)$

\begin{tabular}{|c|c|c|}
\hline Characteristics & & No. of patients $(\%)$ \\
\hline Sex & Female : Male & $4: 14(22.2: 77.8)$ \\
\hline Age & & Median 59.5 years (range $42-83$ ) \\
\hline \multirow[t]{3}{*}{ Hepatitis etiology } & $\mathrm{B}$ & $14(77.8)$ \\
\hline & $\mathrm{C}$ & $1(5.5)$ \\
\hline & nonB/nonC & $3(16.7)$ \\
\hline \multirow[t]{4}{*}{ Previous treatments } & None & $3(16.7)$ \\
\hline & Multiple TACE & $6(33.3)$ \\
\hline & Multiple TACE+RFA & $4(22.2)$ \\
\hline & Multi-modality $^{*}$ & $5(27.8)$ \\
\hline \multirow[t]{2}{*}{ Hepatic segment with recurrence } & Single & $8(44.4)$ \\
\hline & Multiple & $10(55.6)$ \\
\hline \multirow[t]{2}{*}{ Child-Pugh Score } & $\mathrm{A}(5)$ & $17(94.4)$ \\
\hline & $\mathrm{A}(6)$ & $1(5.6)$ \\
\hline $\mathrm{AFP}>9 \mathrm{ng} / \mathrm{ml}$ at RT & & $11(61.1)$ \\
\hline $\mathrm{PIVKA}>35 \mathrm{mIU} / \mathrm{ml}$ at $\mathrm{RT}$ & & $10(55.6)$ \\
\hline Portal vein thrombosis & No & $18(100)$ \\
\hline \multirow[t]{3}{*}{ Number of lesions } & 1 & $15(83.3)$ \\
\hline & 2 & $2(11.1)$ \\
\hline & 3 & $1(5.6)$ \\
\hline Maximum tumor diameter & & Median $1.95 \mathrm{~cm}$ (range 1.0-3.3) \\
\hline Cumulative tumor diameter & & Median $2.05 \mathrm{~cm}$ (range 1.0-4.4) \\
\hline \multirow[t]{4}{*}{ Dose per fraction/total dose } & 9 Gy/36 Gy & $4(22.2)$ \\
\hline & $11 \mathrm{~Gy} / 44 \mathrm{~Gy}$ & $3(16.7)$ \\
\hline & $13 \mathrm{~Gy} / 52 \mathrm{~Gy}$ & $8(44.4)$ \\
\hline & $15 \mathrm{~Gy} / 60 \mathrm{~Gy}^{\dagger}$ & $3(16.7)$ \\
\hline
\end{tabular}

${ }^{*}$ Resection + TACE + RFA $(n=2)$, resection + TACE + sorafenib $(n=1)$, TACE + hepatic arterial chemotherapy + RFA $(n=1)$, and TACE + sorafenib + hepatic arterial chemotherapy $(n=1)$.

"Dose level 4 (15 Gy $\times 4$ fractions) was added after no DLT was observed at level 3.

Abbreviations: RFA $=$ Radiofrequency ablation; TACE $=$ Transarterial chemoembolization; AFP = Alpha-feto protein; PIVKA $=$ Proteins induced by vitamin $\mathrm{K}$ absence or antagonist-II; UICC $=$ International union against cancer.

intrafractional- and interfractional uncertainties, and poor resolution of tumor in x-ray images used for image guidance. A simple method to overcome these limitations is increasing the PTV margins; however, without effective management of liver motion, the volume of non-target liver will increase, hence increasing hepatic toxicity. Abdominal compression is a simple and effective method of reducing diaphragmatic motion. Using gold fiducial markers, Wunderlink et al. showed that abdominal compression was effective in reducing liver tumor motion, yielding small and reproducible excursions in three dimensions [17]. Using rigid liver-to-liver registration of cone beam CT (CBCT) to planning CT, Eccles et al. showed that interfraction liver deformations and GTV displacement in patients undergoing SBRT with abdominal compression were small in most patients [18].
Fiducial markers have the advantage of being visible on x-ray images and fluoroscopy loops, hence increasing targeting accuracy in image-guided SBRT for intrahepatic tumors. However, fiducial placement carries risks specific to markers including migration, additional costs, and imaging artifacts on CT. Procedure-related risks include pain, pneumothorax, hemothorax, perforation of non-target organs, infection, and tumor seeding. These risks can increase substantially with insertion of multiple markers [19]. An alternative is to use the contour of the entire liver as a surrogate. HT uses onboard MVCT for image guidance. Intrahepatic tumors are known to be poorly visible in MVCT as well as in CBCT images. Since installation of HT at our institution in 2006, we have been using rigid liver-to-liver registration of MVCT to planning CT for treatment with HT [20]. Slow acquisition of MVCT 
Table 2: Dosimetric parameters

\begin{tabular}{|c|c|c|}
\hline \multicolumn{2}{|l|}{ PTV volume } & Median 79.9 cc (range 8.2-225.3) \\
\hline \multicolumn{2}{|l|}{ Volume of normal liver } & Median 1124 cc (range $801-1736)$ \\
\hline \multicolumn{2}{|l|}{ Mean dose to normal liver } & Median 9.7 Gy (range 3.0-14.3) \\
\hline \multicolumn{2}{|l|}{ Dose to $700 \mathrm{cc}$ normal liver } & Median 2.65 Gy (range $0.5-10$ ) \\
\hline \multirow[t]{6}{*}{ Volume of normal liver receiving } & $<2.5 \mathrm{~Gy}$ & Median 800 cc (range 208-1233) \\
\hline & $<5.0 \mathrm{~Gy}$ & Median 582 cc (range 160-1074) \\
\hline & $<7.5 \mathrm{~Gy}$ & Median 488 cc (range 131-915) \\
\hline & $<10.0 \mathrm{~Gy}$ & Median 391 cc (range 101-700) \\
\hline & $<12.5 \mathrm{~Gy}$ & Median 311 cc (range 89-533) \\
\hline & $<15.0 \mathrm{~Gy}$ & Median 223 cc (range 65-402) \\
\hline Max dose to bowel/stomach & & Median 14.3 Gy (range 0.1-26.4) \\
\hline Max dose to spinal cord & & Median 11.0 Gy (range 4.8-19.5) \\
\hline
\end{tabular}

Abbreviations: PTV = Planning target volume.

may be a disadvantage; however, abdominal compression reducing breathing motion to $<5 \mathrm{~mm}$ and liver-to-liver registration in the 3-dimensional view improves accuracy of image-guidance [21]. Comparison of pre- and posttreatment setup corrections in the current study has shown MVCT is an adequate tool for image-guidance in treating hepatic tumors (Table 5 and Figure 3 ). Another advantage of HT is its effectiveness in treating multiple targets simultaneously [11], and potential benefits include reduced time for treatment set-up and delivery and increased patient compliance.

Our results suggest that helical IMRT-based SBRT is an effective tool for local control of up to three HCCs targeted simultaneously, provided that an adequate dose

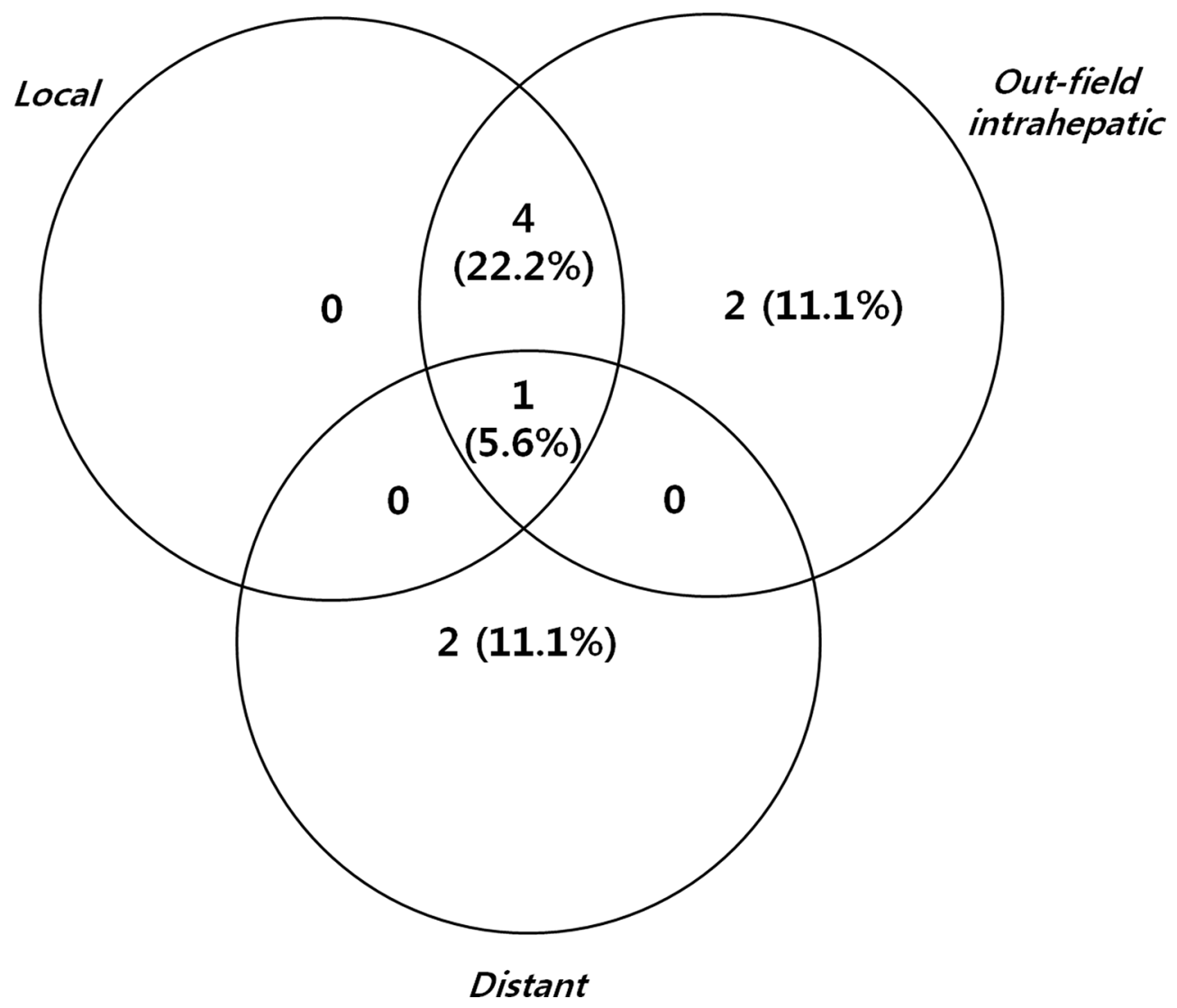

Figure 1: Patterns of all failure. 
Table 3: Treatment related toxicities within 6 months after SBRT

\begin{tabular}{|l|l|l|l|l|}
\hline \multirow{2}{*}{ Toxicity } & \multicolumn{3}{c}{ CTCAE v3.0 grade } \\
\cline { 3 - 5 } \cline { 3 - 5 } & AST & 7 & 0 & $\mathbf{3}$ \\
\hline & ALT & 4 & 0 & 0 \\
\hline & Albumin & 0 & 1 & 0 \\
\hline & ALP & 1 & 0 & 0 \\
\hline & Bilirubin & 0 & 1 & 0 \\
\hline & INR & 1 & 0 & 0 \\
\hline Hematologic & Leukocytes & 6 & $7^{*}$ & $2^{\dagger}$ \\
\hline & Hemoglobin & 1 & 0 & 0 \\
\hline & Platelets & 2 & $1^{\ddagger}$ & $5^{\S}$ \\
\hline Gastrointestinal & Anorexia & 3 & 0 & 0 \\
\hline & Nausea & 1 & 0 & 0 \\
\hline Other & Fatigue & 4 & 1 & 0 \\
\hline & Pain & 1 & 0 & 0 \\
\hline Pulmonary & RT pneumonitis & 6 & 1 & 0 \\
\hline
\end{tabular}

Worsening toxicities due to SBRT are recorded; toxicities due to salvage treatment after treatment failure are not recorded.

${ }^{*}$ Four patients had grade 1 leukocytopenia prior to SBRT.

TWo patients had grade 2 leukocytopenia prior to SBRT.

One patient had grade 1 thrombocytopenia prior to SBRT.

${ }^{\S}$ Four patients had grade 2 and one patient had grade 1 thrombocytopenia prior to SBRT.

Abbreviations: $\mathrm{CTCAE}=$ Common terminology criteria for adverse events; AST $=$ Aspartate Aminotransferase; $\mathrm{ALT}=$ Alanine aminotransferase; ALP = Alkaline phosphatase; INR = International normalization ratio.

A

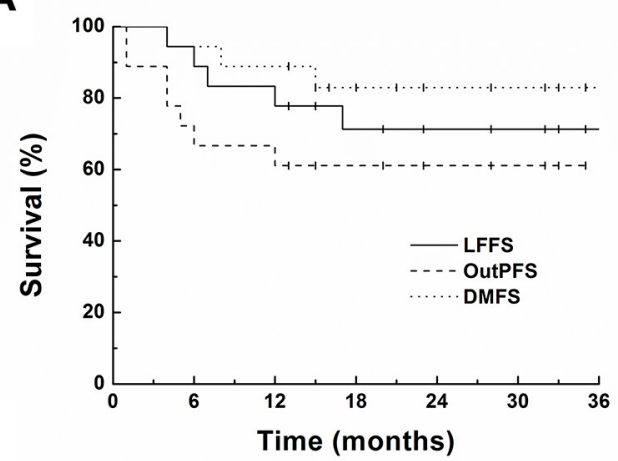

C

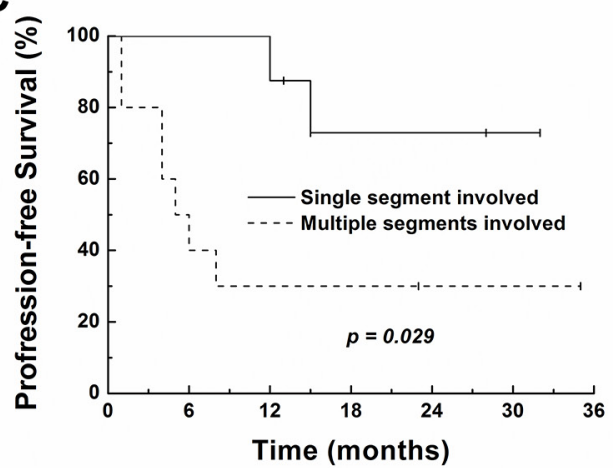

B

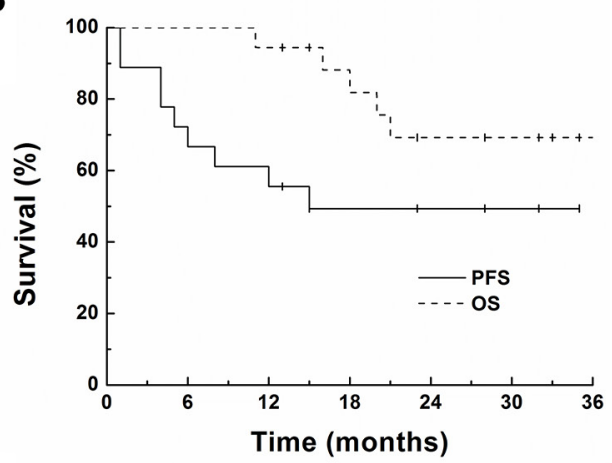

D

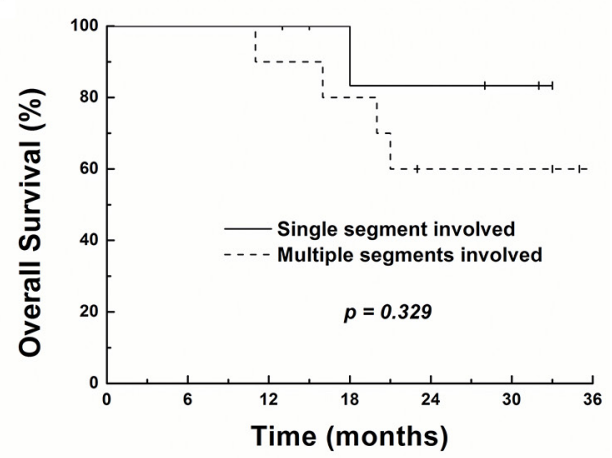

Figure 2: Survival curves showing local failure-free (LFFS), intrahepatic out-field progression-free (OutPFS), distant metastasis-free (DMFS), progression-free (PFS) and overall survival (OS) for all patients (4A and 4B). PFS and OS of the patients with $(n=10)$ and without multi-segment recurrences $(n=8)$ are shown in Figure 4C and 4D. 
Table 4: Factors influencing progression-free survival

\begin{tabular}{|c|c|c|c|c|c|c|c|}
\hline \multirow[b]{2}{*}{ Prognostic factors } & \multirow[b]{2}{*}{ No of Pts (\%) } & \multicolumn{3}{|c|}{ Univariate analysis } & \multicolumn{3}{|c|}{ Multivariate analysis } \\
\hline & & 2-yr PFS (\%) & $95 \%$ CI & $\boldsymbol{P}$ & $\mathbf{R R}$ & $95 \%$ CI & $\boldsymbol{P}$ \\
\hline \multicolumn{8}{|l|}{ Dose level } \\
\hline $3-4$ & $11(61)$ & 63.6 & $35-92$ & 0.209 & 2.425 & $0.47-12.6$ & 0.293 \\
\hline $1-2$ & $7(39)$ & 28.6 & $0-62$ & & & & \\
\hline \multicolumn{8}{|l|}{ Age } \\
\hline$<60$ & $9(50)$ & 44.4 & $12-77$ & 0.580 & & & \\
\hline$\geq 60$ & $9(50)$ & 53.3 & $19-87$ & & & & \\
\hline \multicolumn{8}{|l|}{ Etiology } \\
\hline Other & $4(22)$ & 50.0 & $1-99$ & 0.694 & & & \\
\hline $\mathrm{HBV}$ & $14(78)$ & 49.0 & $22-76$ & & & & \\
\hline \multicolumn{8}{|l|}{ Pre-RT AFP } \\
\hline$<9 \mathrm{ng} / \mathrm{ml}$ & $6(33)$ & 25.0 & $0-65$ & 0.389 & & & \\
\hline$\geq 9 \mathrm{ng} / \mathrm{ml}$ & $12(67)$ & 58.3 & $30-86$ & & & & \\
\hline \multicolumn{8}{|l|}{ Cumul diameter } \\
\hline$<3 \mathrm{~cm}$ & $13(72)$ & 61.5 & $35-87$ & 0.100 & 4.003 & $0.42-38.3$ & 0.229 \\
\hline$\geq 3 \mathrm{~cm}$ & $5(38)$ & 20.0 & $0-55$ & & & & \\
\hline \multicolumn{8}{|l|}{ Multiplicity } \\
\hline No & $15(83)$ & 52.5 & $27-788$ & 0.289 & 0.698 & $0.06-8.7$ & 0.780 \\
\hline Yes & $3(17)$ & 33.3 & $0-87$ & & & & \\
\hline \multicolumn{8}{|l|}{ Multi-segments } \\
\hline No & $8(44)$ & 72.9 & $41-100$ & 0.029 & 8.561 & $1.20-61.3$ & 0.033 \\
\hline Yes & $10(56)$ & 30.0 & $2-58$ & & & & \\
\hline
\end{tabular}

Abbreviations: $\mathrm{RR}=$ Relative risk; $\mathrm{HBV}=$ Hepatitis $\mathrm{B}$ virus.

Table 5: Comparison of pre- and post-treatment setup corrections

\begin{tabular}{|l|c|c|}
\hline \multicolumn{1}{|c|}{ Location of tumor } & $\begin{array}{c}\text { Pre-SBRT displacement } \\
\mathbf{R}^{*} \text { (average } \pm \text { SD, mm) }\end{array}$ & $\begin{array}{c}\text { Post-SBRT displacement } \\
\mathbf{R}^{*} \text { (average } \pm \text { SD, mm) }\end{array}$ \\
\hline Group I (segment 1), $n=1$ & $3.9 \pm 1.2$ & $1.1 \pm 0.7$ \\
\hline Group II (segments 2-4), $n=3$ & $6.3 \pm 2.8$ & $1.5 \pm 1.0$ \\
\hline Group III (segments 5-6), $n=1$ & $7.5 \pm 1.3$ & $2.6 \pm 0.8$ \\
\hline Group IV (segments 7-8), $n=10$ & $5.0 \pm 2.0$ & $1.6 \pm 1.1$ \\
\hline All groups, $n=14$ & $5.4 \pm 2.3$ & $1.6 \pm 1.1$ \\
\hline
\end{tabular}

$* \mathrm{R}=\sqrt{\mathrm{X}^{2}+\mathrm{Y}^{2}+\mathrm{Z}^{2}}$

of radiation is delivered. Another contributing factor for improving local control may be the added margin for microscopic satellite lesions. Clinicopathologic studies have shown that microscopic satellite lesions of $\mathrm{HCC}$ can be detected 5-10 $\mathrm{mm}$ around the gross tumor [22, 23]; thus, providing adequate margins is an important but often overlooked issue. Previous dose escalation studies defined CTV as GTV [12] or ITV [13] without an additional margin, whereas we added a $5 \mathrm{~mm}$ margin to ITV to define CTV in order to cover microscopic satellite lesions.
Extra measures including the use of 4D CT and abdominal compression allowed safe incorporation of the additional margin without increasing PTV substantially.

DLT was not reached even after the dose was escalated to a higher level than initially planned. Our results suggest that a further dose escalation may be possible if eligibility criteria are strictly met. Local control of HCCs, which are relatively radiosensitive, seems achievable at dose level 3; however patients with intrahepatic cholangiocarcinoma and metastatic tumors 


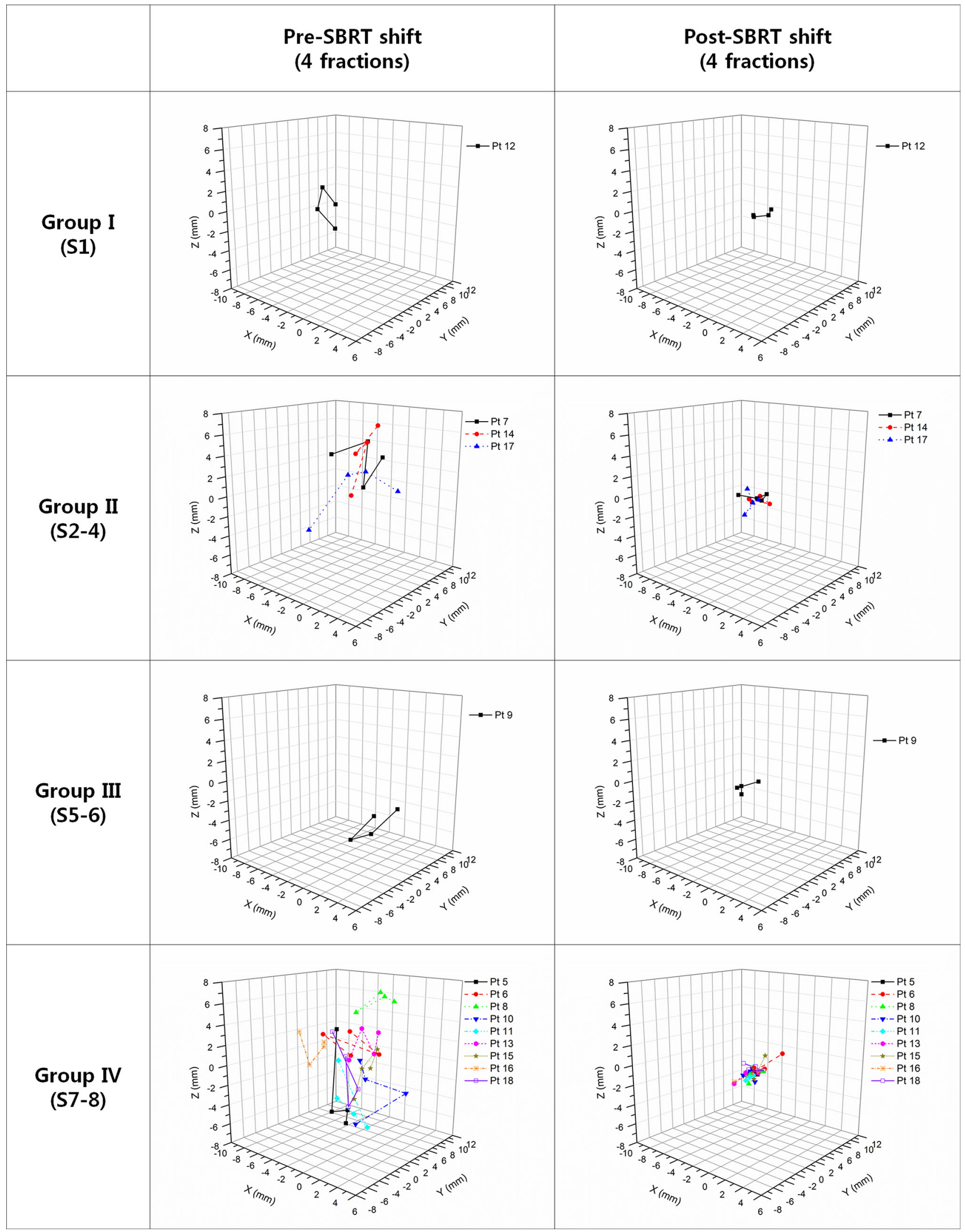

Figure 3: Comparison of pre- and post-treatment setup corrections for $\mathbf{4}$ fractions. Patients are grouped according to tumor location (Group I: segment 1; Group II: segments 2, 3, and 4; Group III: segments 5 and 6; and Group IV: segments 7 and 8). 
such as those from colorectal cancer may require a higher dose for prolonged local control. These patients may benefit from further dose escalation, since the liver function is not compromised by long-term exposure of the liver to viral hepatitis or alcohol consumption.

Use of SBRT for HCC is often initiated only after multiple attempts for local control in curative as well as palliative settings [6]. Many studies have shown the efficacy of SBRT in local control for HCC. Although it seems obvious that patients with multi-segmental recurrences are prone to intrahepatic out-field failures, this is the first prospective study to show that these patients may not be good candidates for SBRT. We suggest that a history of multi-segmental recurrences may need to be an exclusion criterion if SBRT is to be used with a curative aim. Another approach may be to use SBRT in combination with transcatheter arterial chemoembolization (TACE). SBRT may be used for intrahepatic tumors after incomplete treatment with TACE [24], or in combination with TACE or sorafenib for the treatment of multiple HCCs.

The current study is limited by inclusion of mostly small tumors (range 1.0-3.3 cm), and the SBRT protocol needs to be verified for larger tumors. A phase II trial to determine the efficacy of the current SBRT protocol is underway.

Helical IMRT-based SBRT was well-tolerated and showed promising results for adequately selected HCC patients. Exclusion of patients with multi-segmental recurrence prior to SBRT may improve out-field intrahepatic and extrahepatic failure rates.

\section{MATERIALS AND METHODS}

\section{Study end points and eligibility}

The primary end point was toxicity assessment, and the secondary end point was local control rate. A diagnosis of HCC was based on either pathologic confirmation or radiologic findings with an elevated serum level of alphafetoprotein (AFP) (> $400 \mathrm{ng} / \mathrm{mL})$ in patients with a high risk of developing HCC [25].

\section{Eligibility}

The study protocol conforms to the ethical guidelines of the 1975 Declaration of Helsinki, and was approved by our Institutional Review Board (4-2011-0650). All subjects gave informed consent prior to enrollment. All enrolled cases were presented at a multidisciplinary tumor board at our institution. Inclusion criteria were as follows: primary HCC not suitable for surgery because it was technically or medically inoperable or because of the patient's refusal; recurrence after multiple treatment including TACE and RFA; maximum tumor diameter $\leq 5 \mathrm{~cm}$ for a single tumor or the sum of diameters being $\leq 6 \mathrm{~cm}$ for up to 3 lesions; normal liver volume greater than $800 \mathrm{~cm}^{3}$; tumor located at least $1 \mathrm{~cm}$ from the wall of the stomach and/or bowel; no prior radiation therapy to the targeted area; adequate liver function (total bilirubin levels $<3 \mathrm{mg} / \mathrm{dL}$, albumin levels $>2.5 \mathrm{~g} / \mathrm{dl}$, normal prothrombin time (PT) / partial thromboplastin time (PTT), and serum levels of aspartate aminotransferase (AST) and alanine aminotransferase (ALT) less than 3 times the upper limit of normal); adequate renal function (creatinine levels $<1.8 \mathrm{mg} / \mathrm{dL}$ or creatinine clearance $>50 \mathrm{~mL} / \mathrm{min}$ ); adequate hematological function (absolute neutrophil count, ANC $\geq 1500 / \mathrm{mm}^{3}$, platelet counts $\geq 50,000 /$ $\mathrm{mm}^{3}$, hemoglobin level $>9 \mathrm{~g} / \mathrm{dL}$ ), no chemotherapy within 14 days of SBRT; Eastern Cooperative Oncology performance status 0-2; Child-Turcotte-Pugh's Class A or $\mathrm{B}$; and age 20 years or older.

\section{Helical IMRT-based SBRT protocol}

Patients were immobilized using Vac-Lock ${ }^{\mathrm{TM}}$ (CIVCO, Coralville, Iowa) and an abdominal compression device was used to minimize internal organ motion. CT images were acquired over ten respiratory phases, with $1.5 \mathrm{~mm}$ slice thicknesses, under shallow respiration using a 4-dimensional CT simulator (SOMATOM Sensation, Siemens, Munich, Germany). Simulation CT images were fused with images from dynamic CT and MRI in order to aid accurate target delineation. The gross tumor volume (GTV) included all detectable tumors, as determined by dynamic CT and MRI. Internal target volume (ITV) was obtained by summing the GTVs of all respiratory motion phases. In order to incorporate microscopic satellite lesions into the target volume, CTV was defined as ITV plus a $5 \mathrm{~mm}$ margin in all directions. A radial margin of $5 \mathrm{~mm}$ and a craniocaudal margin of $7 \mathrm{~mm}$ were added to the CTV in order to define the planning target volume (PTV) (Figure 4). Helical IMRT-based SBRT planning was performed using a Hi-Art TomoTherapy Planning System (Accuray Inc., Madison, WI). The primary IMRT objectives were to maximize the dose to $95 \%$ of the PTV. Dose constraints included: at least $700 \mathrm{cc}$ of total uninvolved liver receiving less than $15 \mathrm{~Gy}$, less than or equal to $2 / 3$ of the right kidney receiving greater than $15 \mathrm{~Gy}$, maximum spinal cord dose $18 \mathrm{~Gy}$, and maximum dose to the stomach or bowel 24 Gy. All patients were treated every-other-day with on-board MVCT for imageguidance [20]. The entire liver was scanned with MVCT immediately before each fraction of SBRT. The MVCT was aligned with the planning $\mathrm{kVCT}$, with special attention to ensure that tumor-containing hepatic segments were exactly matched. Three dimensional offsets, left-right (x-axis), craniocaudal (y-axis), and antero-posterior (z-axis) offsets were recorded.

\section{Use of MVCT for setup corrections}

Two sets of MVCT scans, immediately before and after SBRT, were acquired for 14 patients (patients 5 to 18). Three dimensional offsets in the left-right 
(x-axis), craniocaudal (y-axis), and antero-posterior (z-axis) directions were recorded. Total displacement (R) was calculated using the equation:

$$
\mathrm{R}=\sqrt{X^{2}+Y^{2}+Z^{2}}
$$

Hepatic segments were divided into 5 groups: segment 1 in Group I $(n=1)$, segments 2,3 , and 4 in Group II $(n=3)$, segments 5 and 6 in Group III $(n=1)$, and segments 7 and 8 in Group IV $(n=9)$ [20]. The mean pre- and post-SBRT total displacement $(\mathrm{R})$ was $3.9 \pm 1.2$ $\mathrm{mm}$ and $1.1 \pm 0.7 \mathrm{~mm}$ for Group I, $6.3 \pm 2.8 \mathrm{~mm}$ and $1.5 \pm 1.0 \mathrm{~mm}$ for Group II, $7.5 \pm 1.3 \mathrm{~mm}$ and $2.6 \pm 0.8 \mathrm{~mm}$ for Group III, $5.0 \pm 2.0 \mathrm{~mm}$ and $1.6 \pm 1.1 \mathrm{~mm}$ for Group $\mathrm{IV}$, and $5.4 \pm 2.3 \mathrm{~mm}$ and $1.6 \pm 1.1 \mathrm{~mm}$ for all patients (Table 5). The MVCT offsets of pre- and post-SBRT are shown in Figure 3.

\section{Dose escalation}

Dose escalation started at 36 Gy (9 Gy/fraction) delivered in four fractions for PTV with a subsequent planned escalation of $2 \mathrm{~Gy} /$ fraction per dose-level. Three patients had to be treated in each dose level with no dose limiting toxicity within 1 month after SBRT before escalation to the next level was permitted. If toxicity occurred, a minimum of six patients were treated at that level.

\section{Evaluation}

Patients were assessed during SBRT and after completion of treatment at 1 month, every 3 months for the first 12 months, and every 6 months thereafter. Dynamic liver CT or MRI was performed at each follow-up. Toxicity was graded using the CTCAE version 3.0. Dose-limiting toxicity (DLT) was defined as grade 3 or greater hepatic, GI toxicity occurring within 1 month of SBRT, or radiation induced liver disease (RILD) requiring treatment in the absence of disease progression within 3 months of SBRT [26]. The Modified Response Criteria In Solid Tumors (mRECIST) was used to evaluate treatment response [27]. Local failure was defined as an in-field recurrence within the high-dose region $(>80 \%$ isodose volume), or mRECIST progressive disease. Outfield recurrence was categorized into intrahepatic and extrahepatic (distant metastasis) recurrences.

\section{Statistical analysis}

Local failure-free (LFFS), outfield intrahepatic failure-free (OFFFS), distant metastasis-free (DMFS), progression-free (PFS), and overall survival (OS) were estimated using the Kaplan-Meier method. Survival rates were defined as the time between the last day of SBRT and the first event. Events were death from any cause for
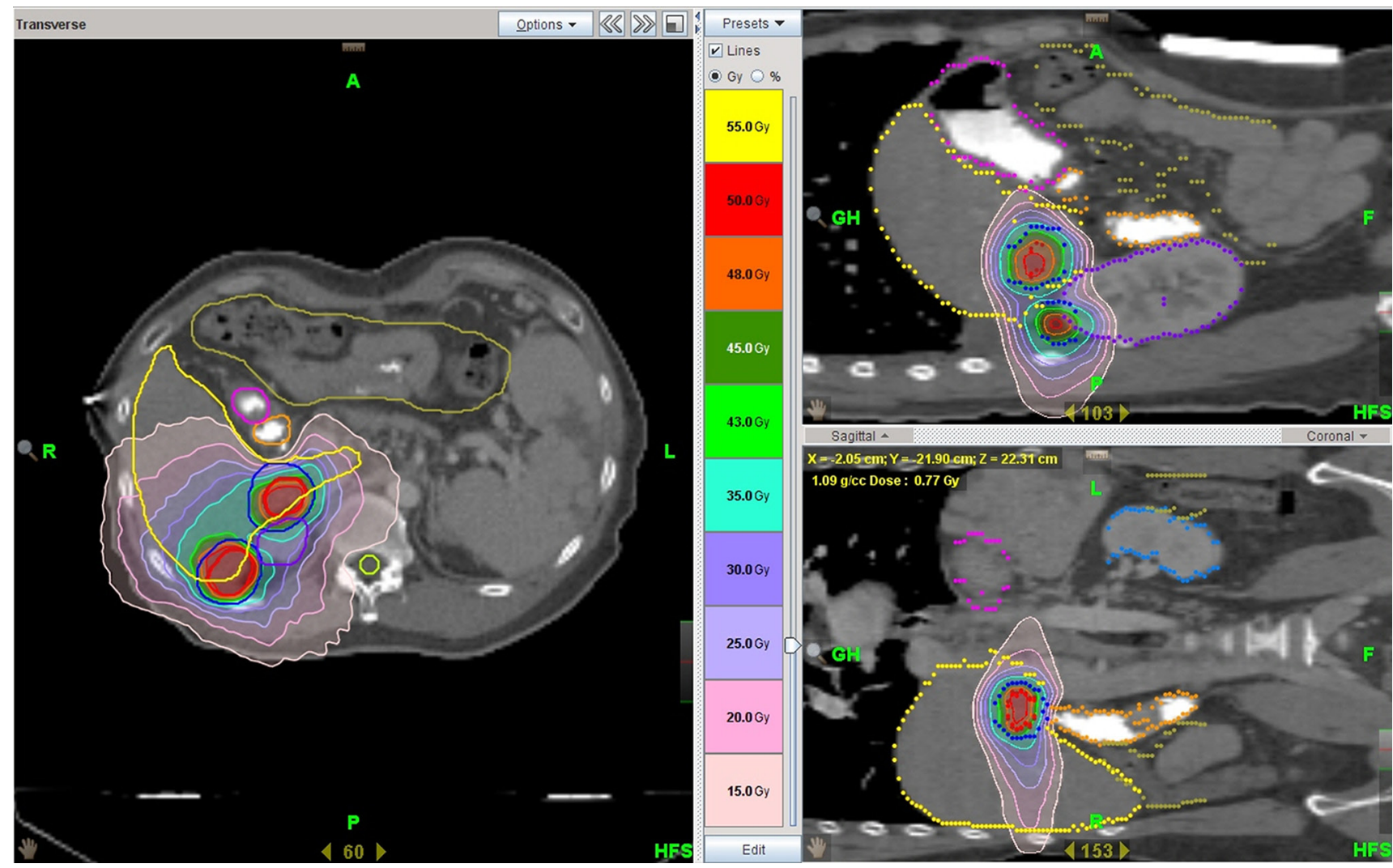

Figure 4: Target volumes and dose distribution for helical IMRT based-SBRT. ITV is shown as a solid red line and PTV as a solid blue line for two target lesions. 
OS, death or tumor progression for PFS, and recurrences as defined above for LFFS, OFFFS, and DMFS. Cox regression analysis was used for multivariate analysis. $P$ values less than 0.05 were considered significant.

\section{ACKNOWLEDGMENTS AND FUNDING}

This study was selected for presentation in an ePoster Discussion session at ASTRO's 2015 Annual Meeting in San Antonio.

This study was supported by a grant from the Korean Health Technology R\&D Project, Ministry of Health and Welfare, Republic of Korea (A121982).

\section{CONFLICTS OF INTEREST}

Conflicts of interest relevant to this article was not reported.

\section{REFERENCES}

1. Korean Liver Cancer Study G, National Cancer Center K. 2014 KLCSG-NCC Korea Practice Guideline for the Management of Hepatocellular Carcinoma. Gut Liver. 2015; 9:267-317.

2. Arii S, Yamaoka Y, Futagawa S, Inoue K, Kobayashi K, Kojiro M, Makuuchi M, Nakamura Y, Okita K, Yamada R. Results of surgical and nonsurgical treatment for smallsized hepatocellular carcinomas: a retrospective and nationwide survey in Japan. The Liver Cancer Study Group of Japan. Hepatology. 2000; 32:1224-1229.

3. Schefter TE, Kavanagh BD, Timmerman RD, Cardenes HR, Baron A, Gaspar LE. A phase I trial of stereotactic body radiation therapy (SBRT) for liver metastases. Int J Radiat Oncol Biol Phys. 2005; 62:1371-1378.

4. Tse RV, Hawkins M, Lockwood G, Kim J, Cummings B, Knox J, Sherman M, Dawson LA. Phase I study of individualized stereotactic body radiotherapy for hepatocellular carcinoma and intrahepatic cholangiocarcinoma. J Clin Oncol. 2008; 26:657-664.

5. Kim YI, Park HC, Lim doH, Kang SW, Han Y, Paik SW. Changes of the liver volume and the Child-Pugh score after high dose hypofractionated radiotherapy in patients with small hepatocellular carcinoma. Radiat Oncol J. 2012; 30:189-196.

6. Lanciano R, Lamond J, Yang J, Feng J, Arrigo S, Good M, Brady L. Stereotactic Body Radiation Therapy for Patients with Heavily Pretreated Liver Metastases and Liver Tumors. Front Oncol. 2012; 2:23.

7. Westerly DC, Schefter TE, Kavanagh BD, Chao E, Lucas D, Flynn RT, Miften M. High-dose MVCT image guidance for stereotactic body radiation therapy. Med Phys. 2012; 39:4812-4819.

8. Nagai A, Shibamoto Y, Yoshida M, Wakamatsu K, Kikuchi Y. Treatment of single or multiple brain metastases by hypofractionated stereotactic radiotherapy using helical tomotherapy. Int J Mol Sci. 2014; 15:6910-6924.

9. Chung Y, Yoon HI, Kim JH, Nam KC, Koom WS. Is helical tomotherapy accurate and safe enough for spine stereotactic body radiotherapy? J Cancer Res Clin Oncol. 2013; 139:243-248.

10. Lee IJ, Seong J, Lee CG, Kim YB, Keum KC, Suh CO, Cho J. Early clinical experience and outcome of helical tomotherapy for multiple metastatic lesions. Int J Radiat Oncol Biol Phys. 2009; 73:1517-1524.

11. Lee TF, Chao PJ, Fang FM, Su TJ, Leung SW, Hsu HC. Helical tomotherapy for single and multiple liver tumours. Radiat Oncol. 2010; 5:58-58.

12. Cárdenes HR, Price TR, Perkins SM, Maluccio M, Kwo P, Breen TE, Henderson MA, Schefter TE, Tudor K, Deluca J, Johnstone PA. Phase I feasibility trial of stereotactic body radiation therapy for primary hepatocellular carcinoma. Clin Transl Oncol. 2010; 12:218-225.

13. Rule W, Timmerman R, Tong L, Abdulrahman R, Meyer J, Boike T, Schwarz RE, Weatherall P, Chinsoo Cho L. Phase I dose-escalation study of stereotactic body radiotherapy in patients with hepatic metastases. Ann Surg Oncol. 2011; 18:1081-1087.

14. Ruchala KJ, Olivera GH, Schloesser EA, Mackie TR. Megavoltage CT on a tomotherapy system. Phys Med Biol. 1999; 44:2597-2621.

15. Stützel J, Oelfke U, Nill S. A quantitative image quality comparison of four different image guided radiotherapy devices. Radiother Oncol. 2008; 86:20-24.

16. Yoon HI, Lee IJ, Han KH, Seong J. Improved oncologic outcomes with image-guided intensity-modulated radiation therapy using helical tomotherapy in locally advanced hepatocellular carcinoma. J Cancer Res Clin Oncol. 2014; 140:1595-1605.

17. Wunderink W, Méndez Romero A, de Kruijf W, de Boer H, Levendag P, Heijmen B. Reduction of respiratory liver tumor motion by abdominal compression in stereotactic body frame, analyzed by tracking fiducial markers implanted in liver. Int J Radiat Oncol Biol Phys. 2008; 71:907-915.

18. Eccles CL, Dawson LA, Moseley JL, Brock K. Interfraction liver shape variability and impact on GTV position during liver stereotactic radiotherapy using abdominal compression. Int J Radiat Oncol Biol Phys. 2011; 80: 938-946.

19. Valentine K, Cabrera T, Roberge D. Implanting metal fiducials to guide stereotactic liver radiation: McGill experience and review of current devices, techniques and complications. Technol Cancer Res Treat. 2014; 13: 253-258.

20. You SH, Seong J, Lee IJ, Koom WS, Jeon BC. Treatment Margin Assessment using Mega-Voltage Computed Tomography of a Tomotherapy Unit in the Radiotherapy of a Liver Tumor. J Korean Soc Ther Radiol Oncol. 2008; 26:280-288. 
21. Brock KK. Imaging and image-guided radiation therapy in liver cancer. Semin Radiat Oncol. 2011; 21: 247-255.

22. Okusaka T, Okada S, Ueno H, Ikeda M, Shimada K, Yamamoto J, Kosuge T, Yamasaki S, Fukushima N, Sakamoto M. Satellite lesions in patients with small hepatocellular carcinoma with reference to clinicopathologic features. Cancer. 2002; 95:1931-1937.

23. Ikeda $\mathrm{K}$, Seki $\mathrm{T}$, Umehara $\mathrm{H}$, Inokuchi $\mathrm{R}$, Tamai $\mathrm{T}$, Sakaida N, Uemura Y, Kamiyama Y, Okazaki K. Clinicopathologic study of small hepatocellular carcinoma with microscopic satellite nodules to determine the extent of tumor ablation by local therapy. Int J Oncol. 2007; 31: 485-491.
24. Kang JK, Cho CK, Yang KM, Yoo HJ, Bae SH, Jung daH, Lee DH, Han CJ, Park SC, Kim YH. Stereotactic body radiation therapy for inoperable hepatocellular carcinoma as a local salvage treatment after incomplete transarterial chemoembolization. Cancer. 2012; 118:5424-5431.

25. Bruix J, Sherman M. Management of hepatocellular carcinoma. Hepatology. 2005; 42:1208-1236.

26. Lawrence TS, Robertson JM, Anscher MS, Jirtle RL, Ensminger WD, Fajardo LF. Hepatic toxicity resulting from cancer treatment. Int J Radiat Oncol Biol Phys. 1995; 31:1237-1248.

27. Lencioni R, Llovet JM. Modified RECIST (mRECIST) assessment for hepatocellular carcinoma. Semin Liver Dis. $2010 ; 30: 52-60$. 